\title{
Logic in first courses for computer science majors
}

\author{
Juris Reinfelds \\ New Mexico State University \\ Las Cruces \\ USA
}

\begin{abstract}
This paper describes the curriculum design of a two-semester introductory course for computer science majors. In each semester the course starts with logic and logic programming. The middle third of the semester continues with functional programming. The last third of the semester is dedicated to imperative programming. In such a presentation sequence reasoning leads to specifications which in turn lead to implementations. The concerns for 'WHAT?' are separated from concerns for 'HOW?'. The advantages and disadvantages of the inclusion of all three major programming paradigms are discussed.
\end{abstract}

Main conference themes: informatics as study topic

Educational areas: higher education,

Study topics: computer science/informatics

Secondary keywords: curriculum development, programming 


\section{INTRODUCTION}

Computer science evolves so rapidly that computer science curricula have to be redesigned more frequently than for any other science. So far three major curriculum recommendations have been published, roughly at ten year intervals. Curriculum 68 [1] defined the scope of the young discipline, but maintained a strong link with numerical mathematics. Curriculum 78 [2] emphasized programming and problem solving as the essential thread which ties together computer science. Curricula 91 [3] emphasized the laboratory aspects of computer science.

Current curriculum research and classroom teaching experiments indicate that 'Curriculum 98', if it happens, will have a strong trend towards the introduction of declarative programming as early as possible in the foundation courses for computer science majors. Harrison [4] has found that the number of universities which include functional programming in their first Computer Science (CS) courses has risen significantly since 1990 and continues to rise at a rapid and increasing rate. Joosten et al. [5] have carried out well designed experiments to determine that the new introductory courses give students a better understanding of more material than the old courses. This is confirmed in a practical way by Lambert, Lindsay and Robertson [6] who come to the same conclusion through informal observations of very large numbers of students who have taken their functional programming course.

Logic programming is the other half. In this paper we will describe a new curriculum which successfully incorporates logic programming, functional programming and imperative programming into a two-semester foundation course sequence for computer science majors. We will discuss the reasons why it is important to include logic and logic programming among the foundation topics of our discipline. We will discuss the major problems introduced by the three-paradigm approach and our solutions. We will discuss the advantages gained. We will conclude with some observations from three years of pilot teaching of our course, first in parallel with, then instead of the conventional monolingual course.

\section{Definitions}

Declarative programming: a declarative program 'declares' what the program should accomplish clearly, unambiguously and precisely, but it leaves to the compiler writer as many of the memory management, process management and other 'how to' decisions as possible. Declarative programming has two branches: logic programming and functional programming. 
Logic programming: uses mathematical logic as the theoretical basis of the language in which a programmer expresses what the program should accomplish. So far it has not been possible to define a programming language using mathematical logic alone. Currently the most widely used logic programming language, Prolog [7], has many 'extralogical' (procedural) extensions. The second generation language Goedel [8] has fewer extralogical extensions. Logic programming has a built-in backtracking mechanism so it is at its best for problems which require systematic searching of the solution space.

Functional programming: builds definitions of functions and uses the computer to evaluate expressions which call these functions. A variable, once bound to a value, retains that value unchanged until the end of calculation. Hence, if an expression has a well defined value, then the order in which a computer may carry out the evaluation steps does not affect the result. The theory of mathematical functions forms the theoretical framework for functional programming.

Imperative (procedural) programming: conventional programming in programming languages like Pascal, Modula, $\mathrm{C}$ or FORTRAN. A program consists of a sequence of commands which describe, step by step, how to manipulate a set of input values to obtain the desired output values.

\section{The role of logic in computer science}

Computer science is the science of information. It studies how to gather, store, manipulate, transform and display information. Problem solving is a key activity. A computer scientist starts with a problem description which is vague and incomplete, manipulates it into a precise and complete specification, transforms the specification into a program which is correct with respect to the specification and runs that program on a computer.

The best way to turn a vague story into a precise specification is to use disciplined reasoning and formal logic. Logic therefore is an essential ingredient of computer science and logic skills should be in every computer scientist's tool box. Until recently it was difficult to include logic into the first course without additional help from coordinated mathematics courses, but Gries and Schneider have used Dijkstra's equational logic to show us in their excellent textbook [9] that logic can be taught and used as an extension of the high school algebra notion that 'equals may be substituted for equals'.

Since logic is central to computer science, we chose to start the first course with a discussion of equational logic and reasoning. The first third of each semester of our course is allocated to the study of problem solving in the framework of logic programming. 


\section{Problem solving patterns}

In all scientific disciplines most problems belong to well defined classes. For each class there are a few well known problem solving patterns and a good solution to a particular problem is obtained by a slight variation of one of these basic problem solving patterns. Upon encountering a new problem the scientist has to recognize to which class it belongs and then choose one of the problem solving patterns of that class. In this way engineers can accurately estimate the time and costs of new buildings, or bridges, or electrical circuit boards or microcomputer chips. New problems which cannot be classified occur infrequently and are assigned to research laboratories.

In computer science we still have a tendency to ignore experience. Computer scientists prefer to solve every problem from first principles as if nothing like it was ever solved before. Our discipline is old enough now to start to develop and teach typical problem solving patterns. We consider the development of a comprehensive set of problem solving patterns as the most important component of our course.

\section{The basic toolbox}

In computer science our basic tools are algorithms, data structures and problem solving patterns. Ever since Wirth [10] published the first definitive set of such algorithms and data structures most computer scientists will agree that their basic toolbox should include algorithms on:

- searching

- sorting

- backtracking

- recursing

- hashing

- parsing

and data structures such as

- arrays, records;

- lists, queues, stacks;

- trees.

By separating our concern for 'What is the essence of the algorithm or data structure?' from the concern for 'How is it implemented?' we can study more algorithms in more depth than in a conventional course, because the conventional course has to present the mathematical structure ('the essence') of the algorithm together with its implementation. 


\section{A wider window on subsequent courses}

A first course should provide a glimpse of the scope of the specialized areas of as many subsequent courses as possible. An extra paradigm extends the scope of the glimpses that can be provided. For example, logic programming with carefully chosen examples introduces the student to data bases, reasoning about programs, expert systems, backtracking problems, parsing problems (compiler courses) and artificial intelligence.

Functional programming leads into executable specifications, algorithm design and analysis, rapid construction of abstract data types, rapid prototyping as well as to theory courses in general. Logic and functional programs are inherently parallel, while imperative programs are sequential unless parallelized with considerable effort.

\section{A THREE PARADIGM FIRST COURSE}

Our first course is a two-semester sequence, four credit hours per semester (three 50 minute lecture periods and one 2 hour laboratory session per week) for a 16 week semester. A strong laboratory component is crucial to the success of the course. Each laboratory session has a prescribed set of objectives. These are explained to the students at the beginning of the laboratory period. Students then work under the supervision of a Teaching Assistant during the rest of the laboratory period, work on their own later and submit a laboratory report for grading before the start of next week's lab. The laboratory reports are graded and returned in the session one week later.

\section{Read before writing}

In each paradigm the students first learn to read, understand and modify relatively large programs which are interesting and well written. In the last laboratory session of each paradigm the students are given a problem description and they have to specify, design, implement and verify their own program which solves the given problem. Experience with our approach by course designers as well as colleagues who taught the course, but were not connected with the curriculum design, has shown that students can reach a sufficient skill level in each of the three paradigms to construct their own programs. The hands-on experience with the comprehension and modification of the given programs is sufficient to provide the students with enough knowledge and confidence to write their own program in the fifth week of each paradigm.

One of the best ways to learn how to express a program in a programming language is to study statement-by-statement dissections of good programs. 
Our lectures and laboratory exercises are built around case studies of program dissections as much as possible. As a consequence our students quickly acquire good comprehension and reasonable application skills in each of the three programming paradigms which we teach in the course.

\section{A spiral approach}

The first semester has 5 weeks devoted to logic programming with emphasis on backtracking, recursing and parsing. The next 5 weeks are spent on functional programming with emphasis on searching, sorting and recursing. The final 5 weeks belong to imperative programming with emphasis on hashing as well as on the efficient implementation of algorithms studied in the previous sections.

The second semester has the same 5-5-5 week split into logic, functional and imperative programming. The first week of each section revises the material taught in the first semester. The remaining weeks develop a deeper understanding of each paradigm through more challenging examples and more sophisticated problem solving patterns.

This approach solves two problems. First, there is the transition period problem when the second semester class will consist of a mixture of students: those who have studied our first course and those who have studied the old one. Depending on the ratio of new to old students and on the overall ability of the class to absorb new material we can allocate more or less emphasis and time to revision of first semester material and so maximize what the students ultimately retain for use in later courses.

Second, the attention span and retention capabilities of most of today's students are such that two smaller chunks of each programming methodology over two semesters provide an opportunity for reinforcement of retained knowledge which is not available if each methodology is studied just once over a longer period of time.

\section{Gradual introduction of new concepts}

Logic programming introduces the essence of declarative programming with fewer and simpler concepts than functional programming. A database containing facts and rules is for mathematically naive students a more intuitive structure than function definitions and currying.

Every separation of concerns is a pedagogical advantage. For example, the typelessness of Prolog is an advantage for the initial five weeks of the first semester. When mixed type and list element management start to get out of hand, students really appreciate the change to the discipline and power of strong typing in Miranda. 


\section{Seamless transition from logical to functional programming}

As the following table shows there is a strong conceptual correspondence between functional and logical programming. This is not surprising because these really are the two faces of declarative programming. A seamless transition ensures that many functional concepts can be built upon logical programming concepts without confusion or relearning.

Table 1 Seamless transition from logic to functional programming

\begin{tabular}{lll}
\hline Concept & Logic Programming & Functional Programming \\
\hline a value & $\begin{array}{l}\text { a constant } \\
\text { a list of constants }\end{array}$ & $\begin{array}{l}\text { a constant (has a type) } \\
\text { a list of constants (same type) } \\
\text { result of function call }\end{array}$ \\
\hline variable & $\begin{array}{l}\text { once bound to a variable, } \\
\text { value cannot be changed, } \\
\text { except on backtracking }\end{array}$ & $\begin{array}{l}\text { once bound to a variable, } \\
\text { the value cannot be changed. } \\
\text { There IS NO backtracking. }\end{array}$ \\
\hline program & database + query & script + expression \\
\hline program execution & for what values of variables & what is the value of the \\
mechanism & does query yield TRUE & expression in its simplest form? \\
\hline program components & facts, rules & function definitions \\
\hline repeated execution & recursion & $\begin{array}{l}\text { recursion } \\
\text { of same program text }\end{array}$ \\
\hline $\begin{array}{l}\text { selection of } \\
\text { alternatives }\end{array}$ & unification & $\begin{array}{l}\text { pattern matching } \\
\text { conditional guards }\end{array}$ \\
\hline Input and Output & extra logical predicates & $\begin{array}{l}\text { input and output modelled } \\
\text { by infinite lists of characters }\end{array}$ \\
\hline
\end{tabular}

\section{Generic versus specific methodology}

New programming languages will come and go, but the method of computation for each major paradigm will remain. We therefore stress the generic nature of logic programming and its model of computation, but we do practical work in the currently most widely used first-generation logic programming language Prolog with its extralogical and procedural extensions. To emphasize generic independence over language choice we are considering the second-generation logic programming language Goedel [9] for the second semester course.

In imperative programming it is essential to study the Dijkstra model [11] of programming, so that one can develop a language independent imperative program structure and reason about the correctness of the program before one commits to code it in full detail in a particular programming language such as $\mathrm{C}$ or Modula, Pascal or $\mathrm{C}++$. 


\section{OUTLINE OF A THREE PARADIGM COURSE}

The complete outline of our course is available in a Technical Report [12]. Here we give, as a sample, an outline of the lectures and laboratories for the first five weeks (logic programming section) of our second semester course. For each week we state the major topics as well as the aim or reason for studying these topics.

\section{Laboratory session outline}

Some of the second semester laboratory sessions build on the work done in one or more first semester laboratories. When this is the case, we will indicate, for example, $271 / 9$ in the margin, which means that this laboratory extends the work of the Week 9 laboratory of CS 271.

Week 1 271/1 UNIX Revision based on 'Test Drive a UNIX Workstation' by J. Bentley. Aim Develop skill of black box testing of laboratory hardware and software systems.

Week 2 271/2-3 Prolog Revision using a labyrinth-search for best path problem.

Aim show strength of Prolog: built-in backtracking mechanism.

Week $3 \quad 271 / 4$ Study and modify Adventure Game, mainly its natural language input part. Aim Introduce parsing and grammars.

Week 4 Study and modify Eliza, the psychiatrist program.

Aim Illustrate problems encountered in grappling with the 'meaning' of text.

Week 5 Students specify, design, implement and test their own logic program.

Problem Open ended problem concerning data base or human interface or both, e.g. Instructor's grade sheet for CS 272.

\section{Lecture outline}

Week 0 (First Lecture) Logic Programming Revision. Ex.: Small restaurant data base.

Week 1 PROLOG: term, clause, rule, unification, search tree, recursion. Ex.: search tree for restaurant problem; stacked alphabet-block problem for recursion.

Aim Develop precise notion of syntax and semantics of Prolog programs.

Week 2 Lists, List Handling; I/O; Negation as failure. Ex.: Graph with loops as a problem solving pattern. Snepscheut's 4-coin problem.

Aim Teach problem solving patterns where logic programming excels.

Week 3 Backtracking. Ex.: HOCUS + POCUS = PRESTO (exhaustive search with generators); Simple Parsing. Ex.: A simple natural language parser.

Aim Demonstrate strength of backtracking; introduce grammars and parsing. 
Week 4 More Parsing. Ex.: Analyze the natural language interface of the Adventure Game (see Lab of Week 3).

Aim Demonstrate the strength and the limits of syntactic analysis.

Week 5 Extra-logical features of Prolog (cut/1, closed world assumption, +/ operator), reasons for their introduction and their weaknesses. If class is a strong group of students, show how Goedel overcomes some of these weaknesses. (FIRST TEST).

Aim To separate the notion of logic programming from first generation programming languages for logic programming.

\section{EXPERIENCE AND CONCLUSIONS}

Each course was first-taught in parallel with the conventional course. In 1993 the faculty of the NMSU CS department decided to teach all sections of both courses in the new way and to accumulate a sufficiently large group of students so that their progress can be monitored over the next 3 to 5 years to assess the effectiveness of the new approach. It has been successfully taught by faculty colleagues who did not participate in the curriculum design. Both faculty and students were very happy with the results. In academic year 94/95 the overall student numbers at NMSU declined slightly, the enrolment in these two courses increased.

Student response has been overwhelmingly positive. We often get unsolicited e-mail from former students who were able to quickly solve a homework problem in some non-CS subject by writing an 'executable specification' in Prolog or Miranda while their classmates had to develop a data structure and implement the algorithm of the solution in an imperative language. Students quickly learn to take advantage of built-in abstract data types (lists especially) and built-in algorithms (backtracking for example).

In conclusion, teacher acceptance and student responses indicate that a multiparadigm, multilanguage approach to introductory computer science teaching is effective and beneficial. With our approach a programming paradigm becomes a problem solving tool instead of being an impediment to a wider view of problem solving patterns as it is after a monolingual course.

\section{ACKNOWLEDGEMENTS}

The author is grateful to the National Science Foundation (NSF) for two curriculum development research grants USE-9150917 and USE-9156218 and NSF Program Director Jim Harri. Special thanks to Dr. M. Auguston who contributed many improvements. Many thanks to the first course discussion 
group, to the teaching assistants, to our system manager and programmer and to our students who put up with cryptic lecture notes and still enjoyed and helped to improve the course.

\section{REFERENCES}

1. ACM Curriculum Committee (1968) Curriculum 68. Comm. ACM 11 (3) pp. 151-197.

2. Austing, R. (ed.) 1979) Curriculum 78. Comm. ACM, 22, (3) pp. 147166.

3. ACM/IEEE-CS Curriculum Task Force (1991) Computing Curricula 1991. IEEE Comp. Soc. Press.

4. Harrison R. (1993) The Use of Functional Languages in Teaching of Computer Science. Journal of Functional Programming 3 (1) pp. 67-75.

5. Joosten, S., van den Berg, K. and van der Hoeven, G. (1993) Teaching Functional Programming to First-Year Students. Journal of Functional Programming 3 (1) pp. 49-65.

6. Lambert, T., Lindsay, P. and Robinson, K., (1993) Using Miranda as a First Programming Language. Journal of Functional Programming 3 (1) pp. 534.

7. Clocksin, W. F. and Mellish, C. S. (1987) Programming in Prolog. Third Edn., Springer Verlag, New York.

8. Hill, P. M. and Lloyd, J. W. (1994) The Goedel Programming Language. MIT Press.

9. Ciries, D. and Schneider, F. B., (1993) A Logical Approach to Discrete Math. Springer Verlag, New York.

10. Wirth, N. (1976) Algorithms + Data Structures = Programs. Prentice Hall.

11. van de Snepscheut, J. L. A. (1993) What Computing is All About. Springer Verlag, New York. 
12. Reinfelds, J. (1994) Curriculum Design for a Three Paradigm First Course. NMSU Computer Science Department Technical Report, NMSUCSTR-9412. 